\title{
THE EXTENSION OF AN ARBITRARY BOOLEAN ALGEBRA TO AN IMPLICATIVE BOOLEAN ALGEBRA
}

\author{
ARTHUR H. COPELAND, SR. AND FRANK HARARY
}

1. Introduction. An implicative Boolean algebra [1] is one which contains an implication or conditional which is appropriate to the theory of probability. The conditional has been defined as an inverse of a cross product operation which is associative, noncommutative, distributive with respect to the binary Boolean operations, satisfies left cancellation, and has the unit element as its unit. It is known that not all Boolean algebras are implicative. In fact it is shown here that any implicative Boolean algebra containing more than two elements is atomless. The question then arises whether there is an essential restriction imposed by introducing such a conditional into an arbitrary Boolean algebra. The question is answered in the negative by the fact that any Boolean algebra can be enlarged so as to include the additional elements produced by the operations of the cross product and the conditional.

The notation and terminology of [1] will be used. Thus T1 to T26 refer to the theorems, $\mathrm{P} 0$ to $\mathrm{P} 6$ to the postulates, and $\cdot, \mathrm{V}, \sim,+, \mathrm{X}$, and $C$ denote conjunction, disjunction, negation, symmetric difference, cross product, and the "if" operation of [1] respectively.

2. Extension. To show that every Boolean algebra $B$ can be extended to an implicative Boolean algebra, we first show that $B$ has a representation in which each nonzero element has the same cardinal power. The cardinality $c(x)$ of an element $x$ is defined as the cardinality of the set of all points of the representation space contained in $x$. Of course $c(x)$ depends on the particular representation. In the following, $B$ is identified with its various representations.

LEMMA 1. Every Boolean algebra $B$ has a representation in which $c(x)=c(1)$ for all $x \neq 0$.

Proof. If $B$ is finite, let $a_{1}, \cdots, a_{n}$ be its atoms. Let the elements $a_{i}$ be represented by pairwise disjoint countably infinite sets of points. In this representation, the cardinality of each nonzero element of $B$ is $\aleph_{0}$.

If $B$ is infinite, let $B$ be represented by the Stone representation

Presented to the Society, December 28, 1951; received by the editors April 22, 1952 and, in revised form, January 30, 1953. 
theorem $[2 ; 3]$. Thus $B$ is a set of subsets of points $\xi$ of a space $S$. We obtain another representation of $B$ by replacing each $\xi$ by a subset $S_{\xi}=\{\xi\} \times S$ of $S \times S$. That is, $S_{\xi}=$ the set of all pairs $(\xi, \eta)$ where $\eta \in S$. Then $c\left(S_{\xi}\right)=c(S)$. In this new representation of $B$, for each nonzero $x \in B, c(x)=c(S)$ since

$$
c(S) \leqq c(x) \leqq c(S \times S)=c(S) \cdot c(S)=c(S) .
$$

The following extension theorem is the principal result of this paper.

Theorem 1. Every Boolean algebra $B$ can be extended to an implicative Boolean algebra $B^{*}$.

Proof. Let $B$ be represented as in Lemma 1 . Let the points $\xi$ of the representation space $S$ be well ordered so that if $\sigma(\xi)$ denotes the lower segment preceding $\xi$, then $c(\sigma(\xi))<c(1)$. This can be done by selecting the least ordinal having the same cardinal power as $S$.

For each subset $x$ of $S$ for which $c(x)=c(S)$, let $T_{x}$ be the unique 1-1 order-preserving mapping of $S$ on $x$. Since $T_{x}$ is 1-1, if $y$ is any subset of $S$ with $c(y)=c(S)$ then $c\left(T_{x}(y)\right)=c(S)$.

The implicative Boolean algebra $B^{*}$ to which we shall extend $B$ is a set of subsets of the above well-ordered series. The Boolean operations are the usual set-theoretic operations. We define $B^{*}$ as the minimal Boolean algebra containing $B$ and such that if $x, y \in B^{*}$, then $z=T_{x}(y) \in B^{*}$ and $w=T_{y}^{-1}(x \cdot y) \in B^{*}$ if $y \neq 0$. Since $c(x)=c(1)$ for all nonzero $x \in B$, it follows inductively that this condition also holds for all nonzero elements of $B^{*}$.

For $x, y \in B^{*}, x, y \neq 0$, we define $x \times y=T_{x}(y)$. We also define $x \times 0=0 \times y=0 \times 0=0$. The above definitions of $B^{*}$ and the cross product imply that $B^{*}$ is closed under this binary operation and hence satisfies postulate P0.

We verify the remaining postulates $\mathrm{P} 1$ to $\mathrm{P} 6$.

P1. $T_{x \times y}(1)=x \times y=T_{x}(y)=T_{x} T_{y}(1)$ which implies $T_{x} T_{y}(\xi)$ $=T_{x \times y}(\xi)$.

Therefore $x \times(y \times z)=T_{x}(y \times z)=T_{x} T_{y}(z)=T_{x \times y}(z)=(x \times y) \times z$.

P3. $x \times(y \cdot z)=T_{x}(y \cdot z)=T_{x}(y) \cdot T_{x}(z)=(x \times y) \cdot(x \times z)$.

P2. (a) $x \times(y \bigvee z)=T_{x}(y \bigvee z)=T_{x}(y) \bigvee T_{x}(z)=(x \times y) \bigvee(x \times z)$.

(b) $T_{x}(y) \bigvee T_{x}(\sim y)=T_{x}(y \bigvee \sim y)=T_{x}(1)=x$.

Multiplying the extreme members of the preceding line by $\sim T_{x}(y)$, we get

(c) $x \cdot \sim T_{x}(y)=T_{x}(\sim y) \cdot \sim T_{x}(y)$.

(d) But $T_{x}(y) \cdot T_{x}(\sim y)=0$ and this implies $T_{x}(\sim y) \cdot \sim T_{x}(y)$ $=T_{x}(\sim y)$. 
Combining the last two steps, we have

(e) $x \cdot \sim T_{x}(y)=T_{x}(\sim y)$, or $x \times \sim y=x \cdot \sim(x \times y)$.

(f) Obviously $x \cdot(x \times y)=x \times y$.

Therefore,

$$
\begin{aligned}
x \times(y+z) & =x \times[(y \cdot \sim z) \vee(z \cdot \sim y)] \\
& =[(x \times y) \cdot(x \times \sim z)] \vee[(x \times z) \cdot(x \times \sim y)] \\
& =[(x \times y) \cdot x \cdot \sim(x \times z)] \vee[(x \times z) \cdot x \cdot \sim(x \times y)] \\
& =[(x \times y) \cdot \sim(x \times z)] \vee[(x \times z) \cdot \sim(x \times y)] \\
& =(x \times y)+(x \times z) .
\end{aligned}
$$

P4. $x \neq 0$ and $T_{x}(y)=T_{x}(z)$ implies $y=z$ since $T_{x}$ is biunique.

Hence $x \neq 0$ and $x \times y=x \times z$ implies $y=z$.

P5. $T_{x}(1)=x \times 1=x$.

P6. If $y \neq 0$, let $z=T_{y}^{-1}(x \cdot y) \in B^{*}$. Then $x \cdot y=T_{y}(z)=y \times z$.

Therefore $B^{*}$ is an implicative Boolean algebra containing $B$ and the theorem is proved. Clearly $B^{*}$ is minimal. The extension on the other hand is not unique. In fact it is possible for two implicative Boolean algebras $B_{1}, B_{2}$ to be isomorphic as Boolean algebras and yet have different cross-product operations.

An example of an implicative Boolean algebra which contains $B$ and is not minimal is as follows. Let $B^{*}$ be a nondenumerable implicative Boolean algebra and let $B$ be any finite Boolean subalgebra. Then $B^{*}$ is not a minimal extension of $B$, for it is shown in the next section that any minimal extension of $B$ is countable.

3. A denumerable implicative Boolean algebra. Consider the situation in which the well-ordered series of the preceding section is the $\omega$-series. Let $0<x_{1}<x_{2}<\cdots$ where the $x_{i}$ are integers and let the sequence of the $x_{i}$ 's be denoted by

$$
x=\left\langle x_{1}, x_{2}, \cdots\right\rangle \text {. }
$$

Let $\xi_{k}=1$ if $k \in x, \xi_{k}=0$ otherwise. Then the sequence $x$ is determined by the sequence of the $\xi_{i}$ 's. Let us denote this sequence by

$$
x=\left(\xi_{1}, \xi_{2}, \cdots\right) \text {. }
$$

By the dyadic number corresponding to sequence $x$ we mean $0 . \xi_{1} \xi_{2} \ldots$. Let $X_{n}=\sum_{k-1}^{n} \xi_{k}$; then the sequence of the $X_{i}$ 's also determines $x$. Let

$$
x=\left[X_{1}, X_{2}, \cdots\right] \text {. }
$$

Thus we have three ways of writing any sequence of positive integers $x_{i}$, each of which determines the other two. Similarly let 


$$
\begin{aligned}
& y=\left\langle y_{1}, y_{2}, \cdots\right\rangle=\left(\eta_{1}, \eta_{2}, \cdots\right)=\left[Y_{1}, Y_{2}, \cdots\right], \\
& z=\left\langle z_{1}, z_{2}, \cdots\right\rangle=\left(\zeta_{1}, \zeta_{2}, \cdots\right)=\left[Z_{1}, Z_{2}, \cdots\right] .
\end{aligned}
$$

Let $v=x \cdot y=$ the set-theoretic intersection of sequences $x, y$. Then

$$
v=x \cdot y=\left(\xi_{1} \eta_{1}, \xi_{2} \eta_{2}, \cdots\right)=\left[V_{1}, V_{2}, \cdots\right] .
$$

The remaining Boolean operations of disjunction, symmetric difference, and negation may also be defined respectively for sequences of integers by

$$
\begin{aligned}
x \vee y & =\left(\xi_{1}+\eta_{1}-\xi_{1} \eta_{1}, \xi_{2}+\eta_{2}-\xi_{2} \eta_{2}, \cdots\right), \\
x+y & =\left(\xi_{1}+\eta_{1}-2 \xi_{1} \eta_{1}, \xi_{2}+\eta_{2}-2 \xi_{2} \eta_{2}, \cdots\right), \\
\sim x & =\left(1-\xi_{1}, 1-\xi_{2}, \cdots\right) .
\end{aligned}
$$

The next binary operation $y \times z$ to be defined for these sequences is given by exactly the same procedure as the formation of $T_{y}(z)$ in Theorem 1:

$$
y \times z=\left\langle y_{z_{1}}, y_{z_{2}}, \cdots\right\rangle .
$$

For the special case of an $\omega$-series, it is possible to write explicitly the "if" operation, $x \subset y$, in the form:

$$
x \subset y=\left(\xi_{y_{1}}, \xi_{y_{2}}, \cdots\right) \text {. }
$$

TheOREM 2. For any sequences of positive integers $x, y$, and $z, x \subset y$ $=z$ if and only if $x \cdot y=y \times z$.

Proof. The proof follows at once from the last two of the following four lemmas. These lemmas may be proved in a straightforward manner by induction and their proofs will be omitted.

LEMMA 2. $X_{x_{n}}=n$.

LEMMA 3. $x_{X_{n}}=n$ if $X_{n}=X_{n-1}+1 ; x_{X_{n}}=x_{X_{n-1}}$ if $X_{n}=X_{n-1}$.

LemMA 4. $V_{n}=Z_{Y_{n}}$ where $z=x \subset y$ and $v=x \cdot y$.

LEMMA 5. $\left\langle y_{z_{1}}, y_{z_{2}}, \cdots\right\rangle=\left[Z_{Y_{1}}, Z_{Y_{2}}, \cdots\right]$.

The set consisting of all increasing infinite sequences of integers and the null sequence does not form an implicative Boolean algebra under the operations defined in this section, for it is not closed under them. However, the set of all sequences whose dyadic numbers are periodic is an implicative Boolean algebra $B$. This includes the numbers 0 and 1 but does not include all dyadic rationals since some rationals fail to conform to the periodicity at the beginning. Further, $B$ is a minimal implicative extension of any finite Boolean algebra 
of more than 2 elements. Also $B$ as a Boolean algebra is isomorphic to the set $C$ of all finite unions of half-open intervals $[a, b)$ such that $0 \leqq a<b \leqq 1$ and $a, b$ are rational.

To indicate the proof of these assertions, we introduce the following notation. Let $(r, n), 0 \leqq r<n$, denote the dyadic number having period $n$, exactly one 1 in each period, and $r 0$ 's preceding the 1 . Then any periodic dyadic number, distinct from zero, is a finite union of numbers $(r, n)$ all having the same $n$. It follows readily from (8) that

$$
(r, n) \times\left(r^{\prime}, n^{\prime}\right)=\left(r+n r^{\prime}, n n^{\prime}\right) .
$$

It is clear that the set $B$ of all periodic dyadic numbers constitutes a Boolean algebra. It is necessary to demonstrate the closure of $B$ under cross product. If $y$ is any periodic dyadic number, then $(r, n)$ $X y$ is readily obtained by using the distributivity of the cross product from the left. It remains to show how to define $y \times(r, n)$. The general definition would require cumbersome notation, but the method can be seen from the following illustration. Let

$$
y=(0,4) \vee(2,4) \vee(3,4) \text { and }(r, n)=(1,2)
$$

Then

$$
\begin{aligned}
y & =y \times 1=y \times[(0,2) \vee(1,2)] \\
& =(0,8) \vee(2,8) \vee(3,8) \vee(4,8) \vee(6,8) \vee(7,8) \text { (by }(10)) .
\end{aligned}
$$

Using (8) we obtain $y \times(1,2)=(2,8) \bigvee(4,8) \bigvee(7,8)$. The element $y=(0,4) \bigvee(2,4) \bigvee(3,4)$ is expanded in order to produce an even number of elements in the disjunction. Then the cross multiplication of the resulting disjunction on the right by $(1,2)$ is equivalent to the selection of the second term in each group of two terms.

We now see that $B$ is closed under cross product. The remaining postulates for an implicative Boolean algebra follow readily from the considerations of this section.

We next show that $B$ as a Boolean algebra is isomorphic to the set $C$ of all finite unions of half-open rational intervals. Since each element of $B$ is a finite disjunction of elements of the form $(r, n)$, it suffices to state the isomorphic correspondence for such elements. But every element of the form $(r, n)$ can be written as a finite disjunction of elements of the form $(\rho, \nu !)$. This follows from

$$
(r, n) \times 1=(r, n) \times \sum_{\alpha=0}^{(n-1) !-1}(\alpha,(n-1) !)=\sum_{\alpha=0}^{(n-1) !-1}(r+\alpha n, n !) .
$$

We may thus give the correspondence for elements of $B$ of the form $(r, n !)$ where $0 \leqq r<n !$. 
Clearly $(r, n !)$ may be written uniquely in the form

$$
(r, n !)=\left(r_{2}, 2\right) \times\left(r_{3}, 3\right) \times \cdots \times\left(r_{n}, n\right), \text { where } 0 \leqq r_{k}<k .
$$

By (10),

$$
r=r_{2}+2 ! r_{3}+3 ! r_{1}+\cdots+(n-1) ! r_{n}
$$

Let

$$
t=\frac{r_{2}}{2 !}+\frac{r_{3}}{3 !}+\cdots+\frac{r_{n}}{n !} .
$$

Then the mapping of $B$ onto $C$ is determined by

$$
(r, n !) \rightarrow\left[t, t+\frac{1}{n !}\right)
$$

The integer $r$ and the rational $t$ may be rewritten in the factorial scale as:

$$
\begin{aligned}
r & =r_{n} r_{n-1} \cdots r_{2} .0, \\
t & =0 . r_{2} r_{3} \cdots r_{n} .
\end{aligned}
$$

The correspondence has been set up in such a way that the interval corresponding to $(r, n !) \times(s, n+1)$ is included in the interval corresponding to $(r, n !)$. From this it follows that inclusion is preserved in general, and hence that the cross product is preserved by the mapping. Clearly the Boolean operations are also preserved. This establishes the isomorphism.

The fact that $B$ is a minimal implicative extension of any finite Boolean algebra of more than 2 elements follows from the consideration of the elements $(0, n),(1, n), \cdots,(n-1, n)$ as the $n$ atoms of a Boolean algebra of $2^{n}$ elements, since any implicative Boolean algebra of more than two elements is atomless by Theorem 3 below.

4. Atomlessness. By definition, an atom $A$ of a Boolean algebra is a nonzero element which does not properly contain any nonzero element. Equivalently, $a$ is an atom if and only if $a \neq 0$ and $a \cdot x=0$ or $a$ for any element $x$.

TheOREM 3. An implicative Boolean algebra $B$ with more than two elements is atomless.

Proof. Assume $a$ is an atom of $B$; then $a \subset b$ is an atom for all $b \neq 0$. For let $x \in B$, then

$x \cdot(a \subset b)=[(b \times x) \subset b] \cdot(a \subset b)=[(b \times x) \cdot a] \subset b$ by T10, T14. 
But $(b \times x) \cdot a=0$ or $a$ since $a$ is an atom. Thus

$$
x \cdot(a \subset b)=0 \text { or } a \subset b .
$$

In particular $a \subset a$ is an atom. But by T17, $a \subset a=1$ which contradicts the hypothesis that $B$ has more than two elements.

We conclude this section with a remark concerning a relation between implicative Boolean algebras and certain prime ideals. Let $a$ denote an atom and $I(a)$ denote the prime ideal of all elements of $B$ not containing $a$. Let $B^{*}$ be any implicative Boolean algebra containing $B$. Then in $B^{*}, x \subset a$ is defined and, as $x$ ranges over $B, x \subset a$ takes on the values 0 or 1 according to whether $x \in \dot{I}(a)$ or not. For $x \subset a$ $=(x \cdot a) \subset a$ by T16 and $x \cdot a=0$ if $x \in I(a),=a$ otherwise; and $a \subset a=1$ by $\mathrm{T} 17$.

5. A probability property. By a probability distribution $p(x)$ on an implicative Boolean algebra $B$, we mean a functional satisfying P7 to P10 of [1].

Theorem 4. Let $B$ and $p(x)$ be as above. If $x_{1}, x_{2}, \cdots, x_{n} \in B$, then there exist $y_{1}, y_{2}, \cdots, y_{n} \in B$ such that the elements $y_{i}$ are independent and $p\left(y_{i}\right)=p\left(x_{i}\right), i=1,2, \cdots, n$.

Proof. If $n=2$ and $x_{1}, x_{2}$ are given, let

$$
y_{1}=x_{1}, \quad y_{2}=\left(x_{1} \times x_{2}\right) \vee\left(\sim x_{1} \times x_{2}\right) \text {. }
$$

Then $y_{1} \cdot y_{2}=x_{1} \cdot\left[\left(x_{1} \times x_{2}\right) \bigvee\left(\sim x_{1} \times x_{2}\right)\right]=\left[x_{1} \cdot\left(x_{1} \times x_{2}\right)\right] \bigvee\left[x_{1} \cdot\left(\sim x_{1} \times x_{2}\right)\right]$ $=x_{1} \times x_{2}$, and $p\left(y_{2}\right)=p\left(x_{1} \times x_{2}\right)+p\left(\sim x_{1} \times x_{2}\right)=p\left(x_{1}\right) p\left(x_{2}\right)+\left(1-p\left(x_{1}\right)\right)$ $p\left(x_{2}\right)=p\left(x_{2}\right)$ by T25, P10, and T24. Thus $p\left(y_{1} \cdot y_{2}\right)=p\left(x_{1} \times x_{2}\right)$ $=p\left(x_{1}\right) p\left(x_{2}\right)=p\left(y_{1}\right) p\left(y_{2}\right)$.

If $n=3$ and $x_{1}, x_{2}, x_{3}$ are given, let

$$
\begin{aligned}
y_{1}= & x_{1}, \quad y_{2}=\left(x_{1} \times x_{2}\right) \vee\left(\sim x_{1} \times x_{2}\right), \\
y_{3}= & {\left[\left(y_{1} \cdot y_{2}\right) \times x_{3}\right] \vee\left[\left(y_{1} \cdot \sim y_{2}\right) \times x_{3}\right] } \\
& \vee\left[\left(\sim y_{1} \cdot y_{2}\right) \times x_{3}\right] \vee\left[\left(\sim y_{1} \cdot \sim y_{2}\right) \times x_{3}\right] .
\end{aligned}
$$

As above $y_{1}, y_{2}$ are independent and $p\left(y_{3}\right)=p\left(x_{3}\right)$. To show $y_{1}, y_{3}$ independent, form $y_{1} \cdot y_{3}=\left[\left(y_{1} \cdot y_{2}\right) \times x_{3}\right] \bigvee\left[\left(y_{1} \cdot y_{2}\right) \times x_{3}\right]$. Thus $p\left(y_{1} \cdot y_{3}\right)$ $=\left[p\left(y_{1} \cdot y_{2}\right)+p\left(y_{1} \cdot \sim y_{2}\right)\right] p\left(x_{3}\right)=p\left(y_{1}\right) p\left(x_{3}\right)=p\left(y_{1}\right) p\left(y_{8}\right)$. The elements $y_{2}, y_{3}$ are also easily shown to be independent. The proof for arbitrary $n$ is similar. ${ }^{1}$

${ }^{1}$ Errata of $[1] ; 1$. Last sentence of $\$ 1$ should read "Moreover ' $x$ strictly implies $y$ ' whenever ' $y$ if $x$ ' is a tautology. ${ }^{n} 2$. Line 13, p. 290 should be $x \times y=v_{\mu_{1}}, v_{\mu_{2}}, \cdots$. 3. Line 21, p. 290 , for " $x$ and $y^{n}$ read " $x$ and $u$." 


\section{REFERENCES}

1. A. H. Copeland, Sr., Implicative Boolean algebra, Math. Zeit. vol. 53 (1950) pp. 285-290.

2. M. H. Stone, The theory of representations of Boolean algebras, Trans. Amer. Math. Soc. vol. 40 (1936) pp. 37-111.

3. Orrin Frink, Jr., Representations of Boolean algebras, Bull. Amer. Math. Soc. vol. 47 (1941) pp. 755-756.

UNIVERSITY OF MICHIGAN 